\title{
UNBOUNDED 2-HYPEREXPANSIVE OPERATORS
}

\author{
ZENON J. JABŁOŃSKI AND JAN STOCHEL \\ Instytut Matematyki, Uniwersytet Jagielloński, ul. Reymonta 4, PL-30059, \\ Kraków, Poland (jablonsk@im.uj.edu.pl; stochel@im.uj.edu.pl)
}

(Received 23 December 1999)

\begin{abstract}
It is shown that most properties of (bounded) completely hyperexpansive operators remain valid for unbounded 2-hyperexpansive operators. Powers of closed 2-hyperexpansive operators are proved to be closed and 2-hyperexpansive. Various parts of spectra of such operators are calculated. 2-hyperexpansive weighted shifts are investigated. Examples of unbounded closed 2-hyperexpansive (2-isometric) operators with invariant dense domains are established.

Keywords: unbounded operators; closedness of powers of operators; 2-hyperexpansive operators; 2-isometries; paranormal operators; weighted shifts

AMS 2000 Mathematics subject classification: Primary 47A05; 47B37

Secondary 47B20; 47B10
\end{abstract}

\section{Introduction}

It is known that some closed operators with invariant domains-like symmetric, hyponormal, paranormal, etc. - are automatically bounded $[\mathbf{9}, \mathbf{1 1}-\mathbf{1 3}]$. On the other hand, there are closed densely defined operators with invariant domains which are unbounded $[\mathbf{1 3}]$. We mention here two classes of such operators, namely nilpotents and idempotents [14]. The aim of the present paper is to show that, similarly to nilpotents and idempotents, closed 2-hyperexpansive operators with invariant domains may be unbounded. Bounded 2-hyperexpansive operators (and, in particular, 2-isometries) have been studied in $[\mathbf{1}-\mathbf{3}, \mathbf{1 6}, \mathbf{1 9}, \mathbf{2 0}]$.

We prove in $\S 3$ that 2-hyperexpansive operators which are paranormal (in particular subnormal) must be isometric. Recall that powers of (closed) paranormal operators are (closed) paranormal $[\mathbf{4 - 6 , 2 3}$. It turns out that the same assertion remains true for 2-hyperexpansive operators (see $\S 4$ ). In $\S 5$ we calculate various parts of spectra of 2-hyperexpansive operators (cf. $[\mathbf{2}, \mathbf{1 6}, \mathbf{2 0}]$ for the bounded case). Only those parts of spectra which are located on the unit circle are not precisely recognized. Section 6 deals with 2-hyperexpansive weighted shifts. It is proved that for every $p>1$, such operators are perturbations of the unilateral shift by compact operators which belong to the Schatten-von Neumann $p$-class. In general, the case of trace class perturbations $(p=1)$ is excluded. However, some one-dimensional perturbations of 2hyperexpansive weighted shifts are shown to be 2-hyperexpansive. Finally, we notice 
that a 2-hyperexpansive operator with invariant domain can be completely characterized by a family of 2-hyperexpansive weighted shifts attached to it (see Proposition 6.7).

\section{Preliminaries}

From now on $\mathbb{D}$ stands for the open unit disc $\{z \in \mathbb{C}:|z|<1\}$ and $\mathcal{H}$ for a complex Hilbert space. By an operator in $\mathcal{H}$ we understand a linear mapping $T: \mathcal{H} \supset \mathcal{D}(T) \rightarrow \mathcal{H}$ defined on a linear subspace $\mathcal{D}(T)$ of $\mathcal{H}$ which is called the domain of $T$. Denote by $\mathcal{N}(T)$ the kernel of $T$ and by $\mathcal{R}(T)$ the range of $T$. Set

$$
\mathcal{D}^{\infty}(T)=\bigcap_{n=1}^{\infty} \mathcal{D}\left(T^{n}\right),
$$

$T_{[k]}=\left.T\right|_{\mathcal{D}\left(T^{k}\right)}$ for $k \geqslant 1$ and $T_{[\infty]}=\left.T\right|_{\mathcal{D}^{\infty}(T)} . \bar{T}$ and $T^{*}$ stand for the closure and the adjoint of $T$, respectively. A subspace $\mathcal{E}$ of $\mathcal{D}(T)$ is said to be a core of $T$ if $\bar{T}=\overline{\left.T\right|_{\mathcal{E}}}$. Denote by $\operatorname{Sp}_{\mathrm{p}}(T), \operatorname{Sp}_{\mathrm{r}}(T), \operatorname{Sp}_{\mathrm{c}}(T)$ and $\operatorname{Sp}_{\mathrm{ap}}(T)$ the point, the residual, the continuous and the approximate point spectrum of $T$, respectively. Let $\operatorname{Sp}(T)$ stand for the spectrum of $T$. The $\mathcal{C}^{*}$-algebra of all bounded operators on $\mathcal{H}$ is denoted by $\boldsymbol{B}(\mathcal{H})$.

Recall that an operator $T$ in $\mathcal{H}$ is called paranormal if $\|T f\|^{2} \leqslant\|f\|\left\|T^{2} f\right\|$ for $f \in$ $\mathcal{D}\left(T^{2}\right)[\mathbf{4}, \mathbf{6}, \mathbf{1 5}, \mathbf{2 4}]$ and hyponormal if $\mathcal{D}(T) \subset \mathcal{D}\left(T^{*}\right)$ and $\left\|T^{*} f\right\| \leqslant\|T f\|$ for $f \in \mathcal{D}(T)$ $[\mathbf{7}, \mathbf{1 5}, \mathbf{2 1}, \mathbf{2 2}]$. It is well known that hyponormal (in particular subnormal) operators are automatically paranormal $[\mathbf{1 5}, \mathbf{2 1}, \mathbf{2 4}]$.

Given an operator $T$ in $\mathcal{H}$, define the graph norms $\|\cdot\|_{T, n}$ on $\mathcal{D}\left(T^{n}\right)$ by

$$
\|f\|_{T, n}^{2}=\sum_{k=0}^{n}\left\|T^{k} f\right\|^{2}, \quad f \in \mathcal{D}\left(T^{n}\right), \quad n \geqslant 0,
$$

and put $\|\cdot\|_{T}=\|\cdot\|_{T, 1}$. Denote by $\tau^{\infty}(T)$ the locally convex topology on $\mathcal{D}^{\infty}(T)$ induced by the family of norms $\left\{\|\cdot\|_{T, n}\right\}_{n=1}^{\infty}$. Our first observation can be proved straightforwardly by induction [23, Proposition 1].

Lemma 2.1. An operator $T$ in $\mathcal{H}$ is closed if and only if $\mathcal{D}\left(T^{n}\right)$ is complete with respect to $\|\cdot\|_{T, n}$ for every $n \geqslant 1$. Moreover, if $T$ is closed, then $\tau^{\infty}(T)$ is complete.

The next result deals with necessary and sufficient conditions for the orthogonal sum of countably many operators to have invariant domain.

Proposition 2.2. Let $T=\bigoplus_{n=1}^{\infty} T_{n}$ be the orthogonal sum of operators $T_{n}$ acting in Hilbert spaces $\mathcal{H}_{n}$.

(i) If $T_{n} \mathcal{D}\left(T_{n}\right) \subseteq \mathcal{D}\left(T_{n}\right)$ and $\left\|T_{n}^{2} f_{n}\right\|^{2} \leqslant c\left\|f_{n}\right\|_{T_{n}}^{2}$ for $f_{n} \in \mathcal{D}\left(T_{n}\right)$ and $n \geqslant 1$ with some $c>0$, then $T \mathcal{D}(T) \subseteq \mathcal{D}(T)$.

(ii) If $T_{n}=\bar{T}_{n}$ for $n \geqslant 1$ and $T \mathcal{D}(T) \subseteq \mathcal{D}(T)$, then $T_{n} \mathcal{D}\left(T_{n}\right) \subseteq \mathcal{D}\left(T_{n}\right)$ and $\left\|T_{n}^{2} f_{n}\right\|^{2} \leqslant$ $c\left\|f_{n}\right\|_{T_{n}}^{2}$ for $f_{n} \in \mathcal{D}\left(T_{n}\right)$ and $n \geqslant 1$ with some $c>0$. 
Proof. (i) Take a vector $f \in \mathcal{D}(T)$. Then $f=\bigoplus_{n=1}^{\infty} f_{n}$ with $f_{n} \in \mathcal{D}\left(T_{n}\right)$ and

$$
\sum_{n=1}^{\infty}\left\|T_{n} f_{n}\right\|^{2}<\infty
$$

By our assumptions $T_{n} f_{n} \in \mathcal{D}\left(T_{n}\right)$ for every $n \geqslant 1$ and

$$
\sum_{n=1}^{\infty}\left\|T_{n}\left(T_{n} f_{n}\right)\right\|^{2} \leqslant c \sum_{n=1}^{\infty}\left\|f_{n}\right\|_{T_{n}}^{2}<\infty
$$

which yields $T f \in \mathcal{D}(T)$.

(ii) Since $T$ is closed, so is the operator $T:\left(\mathcal{D}(T),\|\cdot\|_{T}\right) \rightarrow\left(\mathcal{D}(T),\|\cdot\|_{T}\right)$. By the closed graph theorem there exists $c>0$ such that

$$
\|T f\|_{T}^{2} \leqslant c\|f\|_{T}^{2}, \quad f \in \mathcal{D}(T) .
$$

It is easily seen that the inclusion $T \mathcal{D}(T) \subseteq \mathcal{D}(T)$ implies that $T_{n} \mathcal{D}\left(T_{n}\right) \subseteq \mathcal{D}\left(T_{n}\right)$ for every $n \geqslant 1$. Hence, by $(2.1)$, we have

$$
\sum_{n=1}^{\infty}\left(\left\|T_{n} f_{n}\right\|^{2}+\left\|T_{n}^{2} f_{n}\right\|^{2}\right) \leqslant c \sum_{n=1}^{\infty}\left(\left\|f_{n}\right\|^{2}+\left\|T_{n} f_{n}\right\|^{2}\right), \quad f=\bigoplus_{n=1}^{\infty} f_{n} \in \mathcal{D}(T) .
$$

Substituting $f=f_{n} \in \mathcal{D}\left(T_{n}\right)$ into the above inequality completes the proof.

\section{Basic properties}

In the present paper we investigate a new class of unbounded operators called 2-hyperexpansive ones (cf. $[\mathbf{1}-\mathbf{3}, \mathbf{1 6}, \mathbf{1 9}, \mathbf{2 0}]$ for the bounded case). Below we include its definition as well as definitions of related classes of operators.

We say that an operator $T$ in $\mathcal{H}$ is

(i) 1-hyperexpansive if $\|T f\| \geqslant\|f\|$ for $f \in \mathcal{D}(T)$,

(ii) 1-isometric if $\|T f\|=\|f\|$ for $f \in \mathcal{D}(T)$,

(iii) 2-hyperexpansive if $\left\|T^{2} f\right\|^{2}-2\|T f\|^{2}+\|f\|^{2} \leqslant 0$ for $f \in \mathcal{D}\left(T^{2}\right)$, and

(iv) 2-isometric if $\left\|T^{2} f\right\|^{2}-2\|T f\|^{2}+\|f\|^{2}=0$ for $f \in \mathcal{D}\left(T^{2}\right)$.

It turns out that bounded 2-isometries are completely hyperexpansive, but this is no longer true for 2-hyperexpansive operators (cf. [2] for the terminology and proofs). Let us recall that 1-hyperexpansive operators do not have the approximate point spectrum in the unit disc $\mathbb{D}$.

Lemma 3.1. If $T$ is a 1-hyperexpansive operator in $\mathcal{H}$, then $\mathbb{D} \cap \mathrm{Sp}_{\mathrm{ap}}(T)=\emptyset$.

Proof. Since $\|T f-z f\| \geqslant\|T f\|-|z|\|f\| \geqslant(1-|z|)\|f\|$ for $f \in \mathcal{D}(T)$ and $z \in \mathbb{C}$, the proof is completed. 
It is shown in [16, Lemma 1] that bounded 2-hyperexpansive operators on $\mathcal{H}$ are 1-hyperexpansive. It turns out that a weaker version of this fact remains valid for unbounded operators. For the convenience of the reader we sketch its proof (see also Corollary 6.8 and Remark 6.9).

Lemma 3.2. If $T$ is a 2-hyperexpansive operator in $\mathcal{H}$, then

(i) $T$ is injective,

(ii) $\|T f\|^{2} \geqslant((n-1) / n)\|f\|^{2}$ for $f \in \mathcal{D}\left(T^{n}\right)$ and $n \geqslant 1$,

(iii) $\|T f\| \geqslant\|f\|$ for $f \in \mathcal{D}^{\infty}(T)$,

(iv) $\left\|T^{n} f\right\|^{2}+(n-1)\|f\|^{2} \leqslant n\|T f\|^{2}$ for $f \in \mathcal{D}\left(T^{n}\right)$ and $n \geqslant 1$, and

(v) $\lim _{n \rightarrow \infty}\left\|T^{n} f\right\|^{1 / n}=1$ for $f \in \mathcal{D}^{\infty}(T) \backslash\{0\}$.

Proof. If $f \in \mathcal{N}(T) \subseteq \mathcal{D}^{\infty}(T)$, then

$$
0 \leqslant\|f\|^{2}=\left\|T^{2} f\right\|^{2}-2\|T f\|^{2}+\|f\|^{2} \leqslant 0,
$$

so (i) is proved. Using once more the fact that $T$ is 2-hyperexpansive, we get

$$
\left\|T^{2} f\right\|^{2}-\|T f\|^{2} \leqslant\|T f\|^{2}-\|f\|^{2}, \quad f \in \mathcal{D}\left(T^{2}\right) .
$$

Replacing $f$ by $T^{k} f$ leads to

$$
\left\|T^{k+2} f\right\|^{2}-\left\|T^{k+1} f\right\|^{2} \leqslant\left\|T^{k+1} f\right\|^{2}-\left\|T^{k} f\right\|^{2}, \quad f \in \mathcal{D}\left(T^{k+2}\right), \quad k \geqslant 0 .
$$

Hence

$$
\begin{aligned}
0 \leqslant\left\|T^{n} f\right\|^{2} & =\sum_{j=1}^{n}\left(\left\|T^{j} f\right\|^{2}-\left\|T^{j-1} f\right\|^{2}\right)+\|f\|^{2} \\
& \leqslant n\left(\|T f\|^{2}-\|f\|^{2}\right)+\|f\|^{2} \\
& =n\|T f\|^{2}+(1-n)\|f\|^{2}, \quad f \in \mathcal{D}\left(T^{n}\right), \quad n \geqslant 1,
\end{aligned}
$$

which implies (ii) and (iv). Letting $n \rightarrow \infty$ in (ii) yields (iii) (clearly (iii) $\Rightarrow$ (i)).

(v) Take $f \in \mathcal{D}^{\infty}(T) \backslash\{0\}$. It follows from (iv) that $\lim _{\sup _{n \rightarrow \infty}}\left\|T^{n} f\right\|^{1 / n} \leqslant 1$. However, according to (iii), the sequence $\left\{\left\|T^{n} f\right\|\right\}_{n=0}^{\infty}$ is monotonically increasing, so

$$
\liminf _{n \rightarrow \infty}\left\|T^{n} f\right\|^{1 / n} \geqslant \lim _{n \rightarrow \infty}\|f\|^{1 / n}=1,
$$

which completes the proof.

It is easily seen that if $T$ is a 2-isometry, then part (iv) of Lemma 3.2 takes the form $\left\|T^{n} f\right\|^{2}+(n-1)\|f\|^{2}=n\|T f\|^{2}$ for $f \in \mathcal{D}\left(T^{n}\right)$ and $n \geqslant 1$. Part (v) of Lemma 3.2 is related to $\left[\mathbf{2 3}\right.$, Lemma 8], where the existence of $\lim _{n \rightarrow \infty}\left\|T^{n} f\right\|^{1 / n} \in[0, \infty]$ has been proved for paranormal operators $T$. 
Corollary 3.3. If $T$ is a 2-hyperexpansive operator in $\mathcal{H}$, then for each $a>1$ and for each $f \in \mathcal{D}^{\infty}(T)$ there exists $c>0$ such that $\left\|T^{n} f\right\| \leqslant c a^{n}$ for all $n \geqslant 0$.

Corollary 3.3 says that every $\mathcal{C}^{\infty}$-vector of a 2-hyperexpansive operator is bounded. This means that unbounded closed 2-hyperexpansive operators with invariant domains (see Example 6.4 below) form a new class of operators for which the answer to the question (i) in the abstract of [23] is in the negative.

We now show that the class of 2-hyperexpansive operators has 'small' intersections with some other known classes of operators. Let us begin with paranormals and their adjoints (amongst them there are seminormal and, in particular, subnormal operators). It is proved in [20, Proposition 4] that if $T \in \boldsymbol{B}(\mathcal{H})$ is completely hyperexpansive (consequently, 2-hyperexpansive) and subnormal, then $T$ is an isometry. It turns out that this assertion remains true for unbounded 2-hyperexpansive operators.

Theorem 3.4. Let $T$ be a densely defined 2-hyperexpansive operator in $\mathcal{H}$ with invariant domain. If $T$ is paranormal, then $\bar{T}$ is an isometry. If $T$ is bounded and $T^{*}$ is paranormal, then $\bar{T}$ is unitary.

Proof. If $T$ is paranormal, then by [23, Theorem 10] and Lemma 3.2 (v) $T$ is bounded and $\|T\| \leqslant 1$ (see also Remark 6.9). In the case where $T$ is bounded and $T^{*}$ is paranormal, the spectral radius of $T^{*}$ is equal to $\left\|T^{*}\right\|=\|T\|[\mathbf{6}$, Theorem 1]; however, the spectrum of $\bar{T}$ is contained in $\overline{\mathbb{D}}$ (because $\bar{T}$ is 2-hyperexpansive, cf. [16, Lemma 1]), so $\operatorname{Sp}\left(T^{*}\right) \subseteq \overline{\mathbb{D}}$. Hence again $\|T\| \leqslant 1$. Applying Lemma 3.2 (iii) to both cases we get

$$
\|f\| \leqslant\|T f\| \leqslant\|T\|\|f\| \leqslant\|f\|, \quad f \in \mathcal{D}(T),
$$

which means that $\bar{T}$ is an isometry. The proof is completed by showing that if $V \in \boldsymbol{B}(\mathcal{H})$ is an isometry and $V^{*}$ is paranormal, then $V$ is unitary. Substituting $f=V h, h \in \mathcal{H}$, into the inequality $\left\|V^{*} f\right\|^{2} \leqslant\|f\|\left\|V^{* 2} f\right\|$, we get $\|h\| \leqslant\left\|V^{*} h\right\|$ for $h \in \mathcal{H}$, so $\mathcal{N}\left(V^{*}\right)=\{0\}$ or, equivalently, $\mathcal{R}(V)=\mathcal{H}$.

Recall that an operator $T$ in $\mathcal{H}$ with invariant domain is said to be nilpotent (respectively, idempotent) if $T^{2} f=0$ (respectively, $T^{2} f=T f$ ) for every $f \in \mathcal{D}(T)[\mathbf{1 3}, \mathbf{1 4}]$. Notice that, contrary to the case of idempotents, powers of closed nilpotents need not be closed. Comparing those operators with 2-hyperexpansive ones, we see that

(i) if $T$ is a 2-hyperexpansive nilpotent, then, by Lemma $3.2(\mathrm{i}), \mathcal{N}(T)=\{0\}$ and consequently $\mathcal{D}(T)=\mathcal{N}\left(T^{2}\right)=\{0\}$, and

(ii) if $T$ is a 2-hyperexpansive idempotent, then once more by Lemma 3.2 (i) $\mathcal{R}(1-T)=$ $\mathcal{N}(T)=\{0\}$, so $T$ is the identity operator on $\mathcal{D}(T)$.

According to $[\mathbf{2 0}$, Remark 3] every 2-hyperexpansive operator $T \in \boldsymbol{B}(\mathcal{H})$ whose range is dense in $\mathcal{H}$ is unitary. In other words, invertible elements of $\boldsymbol{B}(\mathcal{H})$ which are 2hyperexpansive must be unitary. In fact, this assertion remains true if we relax the boundedness of $T$. 
Proposition 3.5. Let $T$ be a densely defined 2-hyperexpansive operator in $\mathcal{H}$ with invariant domain. If one of the following two conditions holds true

(i) $\mathcal{D}(T)=\mathcal{R}(T)$,

(ii) $T$ is closable and $\overline{\mathcal{R}(T)}=\mathcal{H}$,

then $\bar{T}$ is unitary.

Proof. Since $\|T(T f)\|^{2} \leqslant 2\|T f\|^{2}$ for $f \in \mathcal{D}(T)$, we get $\|T h\| \leqslant \sqrt{2}\|h\|$ for $h \in \mathcal{R}(T)$, which means that the operator $\left.T\right|_{\mathcal{R}(T)}$ is bounded and $\left.T\right|_{\mathcal{R}(T)} \subseteq T$. In both cases we conclude that $\bar{T}=\overline{\left.T\right|_{\mathcal{R}(T)}} \in \boldsymbol{B}(\mathcal{H})$ is a 2-hyperexpansive operator with a dense range. Hence, by [20, Remark 3], $\bar{T}$ is unitary.

We conclude this section with two observations based on spectral arguments.

Remark 3.6. If $T \in \boldsymbol{B}(\mathcal{H})$ is a 2-hyperexpansive operator which is not unitary, then, for every $\lambda \in \mathbb{C} \backslash\{0\}, T+\lambda$ is not a 2-hyperexpansive operator. The assertion follows from the fact that if $T \in \boldsymbol{B}(\mathcal{H})$ is 2-hyperexpansive and not unitary, then $\operatorname{Sp}(T)=\overline{\mathbb{D}}$ (cf. [20, Remark 3]). The same reasoning shows that if $T \in \boldsymbol{B}(\mathcal{H})$ is a 2-hyperexpansive operator, then for every $\lambda \in \mathbb{C},|\lambda| \neq 1, \lambda T$ is not a 2-hyperexpansive operator.

\section{Graph norms, closedness and invariant domains}

In this section we investigate the powers of 2-hyperexpansive operators as well as 2hyperexpansive operators with invariant domains. We begin with the description of relationships between graph norms of 2-hyperexpansive operators.

Lemma 4.1. If $T$ is a 2-hyperexpansive operator in $\mathcal{H}$, then

(i) the norms $\|\cdot\|_{T}$ and $\|\cdot\|_{T, n}$ are equivalent on $\mathcal{D}\left(T^{n}\right)$ for $n \geqslant 1$,

(ii) the norms $\|\cdot\|_{T^{n}}$ and $\|\cdot\|_{T, n}$ are equivalent on $\mathcal{D}\left(T^{n+1}\right)$ for $n \geqslant 1$,

(iii) the norms $\|\cdot\|_{T^{n}}$ and $\|\cdot\|_{T, n}$ are equivalent on $\mathcal{D}\left(T^{n}\right)$ for $n \geqslant 1$, provided $T$ is 1-hyperexpansive,

(iv) $\tau^{\infty}(T)$ coincides with the topology induced by $\|\cdot\|_{T}$ on $\mathcal{D}^{\infty}(T)$, and

(v) $T_{[\infty]}$ is $\|\cdot\|_{T}$-bounded,

Proof. (i) By Lemma 3.2 (iv), $\left\|T^{k} f\right\|^{2} \leqslant k\|T f\|^{2}$ for $f \in \mathcal{D}\left(T^{k}\right)$ and $k \geqslant 1$. This in turn implies that

$$
\|f\|_{T, n}^{2} \leqslant\|f\|^{2}+(1+2+\cdots+n)\|T f\|^{2} \leqslant \frac{1}{2} n(n+1)\|f\|_{T}^{2}, \quad f \in \mathcal{D}\left(T^{n}\right), \quad n \geqslant 1 .
$$


(ii) It follows from Lemma 3.2 (ii) that $\|f\|^{2} \leqslant 2\|T f\|^{2}$ for $f \in \mathcal{D}\left(T^{2}\right)$, hence that $\left\|T^{j} f\right\|^{2} \leqslant 2^{n-j}\left\|T^{n} f\right\|^{2}$ for $f \in \mathcal{D}\left(T^{n+1}\right)$ and $0 \leqslant j \leqslant n$, and finally that

$$
\|f\|_{T, n}^{2} \leqslant\|f\|^{2}+\left(2^{n-1}+\cdots+2^{0}\right)\left\|T^{n} f\right\|^{2} \leqslant\left(2^{n}-1\right)\|f\|_{T^{n}}^{2}, \quad f \in \mathcal{D}\left(T^{n+1}\right) .
$$

Since $\|\cdot\|_{T^{n}}^{2} \leqslant\|\cdot\|_{T, n}^{2}$, (ii) is proved.

Condition (iii) can be proved in the same way as (ii). Condition (iv) follows from (i).

(v) Since $\left\|T^{2} f\right\|^{2} \leqslant 2\|T f\|^{2}$ for $f \in \mathcal{D}\left(T^{2}\right)$, we obtain $\|T f\|_{T}^{2} \leqslant 3\|f\|_{T}^{2}$ for $f \in \mathcal{D}\left(T^{2}\right)$, which proves (v) (see also [23, Proposition 2]).

Proposition 4.2. If $T$ is a 2-hyperexpansive operator in $\mathcal{H}$, then

(i) $T^{n}$ is 2-hyperexpansive for $n \geqslant 1$,

(ii) $T^{n}$ is closed for $n \geqslant 1$ provided $T$ is closed and 1-hyperexpansive, and

(iii) $\left.T^{n}\right|_{\mathcal{D}^{\infty}(T)}$ is closed for $n \geqslant 1$ provided $T$ is closed.

Proof. (i) Take $f \in \mathcal{D}\left(T^{2 n}\right)$. The definition of 2-hyperexpansivity enables us to write the following inequalities

$$
\begin{aligned}
1\left(\left\|T^{2} f\right\|^{2}-2\|T f\|^{2}+\|f\|^{2}\right) & \leqslant 0, \\
2\left(\left\|T^{3} f\right\|^{2}-2\left\|T^{2} f\right\|^{2}+\|T f\|^{2}\right) & \leqslant 0, \\
\vdots & \\
(n-1)\left(\left\|T^{n} f\right\|^{2}-2\left\|T^{n-1} f\right\|^{2}+\left\|T^{n-2} f\right\|^{2}\right) & \leqslant 0, \\
n\left(\left\|T^{n+1} f\right\|^{2}-2\left\|T^{n} f\right\|^{2}+\left\|T^{n-1} f\right\|^{2}\right) & \leqslant 0, \\
(n-1)\left(\left\|T^{n+2} f\right\|^{2}-2\left\|T^{n+1} f\right\|^{2}+\left\|T^{n} f\right\|^{2}\right) & \leqslant 0, \\
\vdots & \\
2\left(\left\|T^{2 n-1} f\right\|^{2}-2\left\|T^{2 n-2} f\right\|^{2}+\left\|T^{2 n-3} f\right\|^{2}\right) & \leqslant 0, \\
1\left(\left\|T^{2 n} f\right\|^{2}-2\left\|T^{2 n-1} f\right\|^{2}+\left\|T^{2 n-2} f\right\|^{2}\right) & \leqslant 0 .
\end{aligned}
$$

Summing all the above inequalities yields (i).

Condition (ii) follows from Lemma 2.1 and Lemma 4.1 (iii).

(iii) Since $T$ is closed, we conclude from Lemma 2.1 and Lemma 4.1 (iv) that $T_{[\infty]}$ is closed. By Lemma 3.2 (iii), $T_{[\infty]}$ is 1-hyperexpansive, so (ii) implies that the operator $\left.T^{n}\right|_{\mathcal{D} \infty(T)}=T_{[\infty]}^{n}$ is closed.

An inspection of the proof of Proposition 4.2 (i) shows that if $T$ is a 2-isometry, then so is $T^{n}$ for every $n \geqslant 1$.

It is easy to check that if $T$ is a 2-hyperexpansive operator in $\mathcal{H}$, then $T_{[k]}, k \geqslant 1$, and $T_{[\infty]}$ are 2-hyperexpansive operators in $\mathcal{H}$; if, moreover, $T=T_{[\infty]}$, then $T$ is 2hyperexpansive in pre-Hilbert spaces $\left(\mathcal{D}(T),\|\cdot\|_{T, k}\right)$ and $\left(\mathcal{D}(T),\|\cdot\|_{T^{k}}\right)$ for $k \geqslant 0$. These facts will be exploited below. Similar assertions can be formulated for 2-isometries and 1hyperexpansive operators. In addition, if $T=T_{[\infty]}$ is 1-hyperexpansive in $\mathcal{H}$ and $k \geqslant 1$, 
then $T$ is a 1 -isometry in $\mathcal{H}$ if and only if $T$ is a 1 -isometry in the pre-Hilbert space $\left(\mathcal{D}(T),\|\cdot\|_{T, k}\right)$ (respectively, $\left(\mathcal{D}(T),\|\cdot\|_{T^{k}}\right)$ ).

Theorem 4.3. If $T$ is a closed 2-hyperexpansive operator in $\mathcal{H}$, then

(i) $T_{[k]}$ is closed and 2-hyperexpansive for $k \geqslant 1$,

(ii) $T_{[\infty]}$ is closed and 2-hyperexpansive,

(iii) $(I-z T) \mathcal{D}^{\infty}(T)=\mathcal{D}^{\infty}(T)$ for $z \in \mathbb{D}$, and

(iv) $(I-z T) \mathcal{D}^{\infty}(T)$ is a closed subspace of $\mathcal{H}$ for $z \in \mathbb{C} \backslash \overline{\mathbb{D}}$.

Proof. (i) By Lemma 2.1, the normed space $\left(\mathcal{D}\left(T^{k}\right),\|\cdot\|_{T, k}\right)$ is complete. However, according to Lemma $4.1(\mathrm{i})$, the norms $\|\cdot\|_{T, k}$ and $\|\cdot\|_{T}$ are equivalent on $\mathcal{D}\left(T^{k}\right)$. Hence $\left(\mathcal{D}\left(T^{k}\right),\|\cdot\|_{T}\right)$ is complete, which means that $T_{[k]}$ is closed.

Condition (ii) follows from Proposition 4.2 (iii) with $n=1$.

(iii) In view of (ii) and Lemma $4.1(\mathrm{v})$, the operator $T_{[\infty]}$ is bounded and 2-hyperexpansive as an operator acting on the Hilbert space $\left(\mathcal{D}^{\infty}(T),\|\cdot\|_{T}\right)$. Consequently, the spectrum of $T_{[\infty]}$ calculated in the algebra $\boldsymbol{B}\left(\mathcal{D}^{\infty}(T),\|\cdot\|_{T}\right)$ is contained in $\overline{\mathbb{D}}[\mathbf{1 6}$, Lemma 1], which means that $\left.(I-z T)\right|_{\mathcal{D}^{\infty}(T)}$ is invertible in $\boldsymbol{B}\left(\mathcal{D}^{\infty}(T),\|\cdot\|_{T}\right)$ for $z \in \mathbb{D}$.

(iv) It follows from Lemma 3.2 (iii) and Lemma 3.1 that $\mathbb{D} \cap \operatorname{Sp}_{\text {ap }}\left(T_{[\infty]}\right)=\emptyset$. However, according to (ii), $T_{[\infty]}$ is closed. Hence, if $z \in \mathbb{D}$, then $\left(T_{[\infty]}-z\right)^{-1}$ is closed and bounded, which in turn implies that $(T-z) \mathcal{D}^{\infty}(T)=\mathcal{D}\left(\left(T_{[\infty]}-z\right)^{-1}\right)$ is a closed subspace of $\mathcal{H}$.

We now concentrate on 2-hyperexpansive operators whose successive domains are equal.

Proposition 4.4. If $T$ is a closed 2-hyperexpansive operator in $\mathcal{H}$ and $n \geqslant 1$, then the following conditions are equivalent:

(i) $\mathcal{D}\left(T^{n}\right)=\mathcal{D}\left(T^{n+1}\right)$,

(ii) $\mathcal{D}\left(T^{n}\right)=\mathcal{D}^{\infty}(T)$, and

(iii) $\mathcal{D}\left(T^{n+1}\right)$ is a core of $T_{[n]}$.

Proof. Implications (i) $\Rightarrow$ (ii) $\Rightarrow$ (iii) are obvious.

(iii) $\Rightarrow$ (i) According to Theorem $4.3(\mathrm{i})$, the normed space $\left(\mathcal{D}\left(T^{n+1}\right),\|\cdot\|_{T}\right)$ is complete, so $\mathcal{D}\left(T^{n+1}\right)$ is $\|\cdot\|_{T^{-}}$-closed and simultaneously $\|\cdot\|_{T^{-}}$dense in $\mathcal{D}\left(T^{n}\right)$. Hence, $\mathcal{D}\left(T^{n}\right)=$ $\mathcal{D}\left(T^{n+1}\right)$, which completes the proof.

Closed 2-hyperexpansive operators with invariant domains can be characterized as follows.

Theorem 4.5. If $T$ is a closed 2-hyperexpansive operator in $\mathcal{H}$, then the following conditions are equivalent:

(i) $T \mathcal{D}(T) \subseteq \mathcal{D}(T)$, 
(ii) $\mathcal{D}\left(T^{2}\right)$ is a core of $T$,

(iii) $\mathcal{D}\left(T^{n}\right)$ is a core of $T$ for some $n \geqslant 2$,

(iv) $\mathcal{D}\left(T^{n}\right)$ is a core of $T$ for every $n \geqslant 2$,

(v) $\mathcal{D}^{\infty}(T)$ is a core of $T$, and

(vi) $\mathcal{D}(T)=\mathcal{D}^{\infty}(T)$.

Proof. Implications (i) $\Rightarrow$ (vi) $\Rightarrow$ (v) $\Rightarrow$ (iv) $\Rightarrow$ (iii) $\Rightarrow$ (ii) are obvious. Implication (ii) $\Rightarrow$ (i) follows from Proposition 4.4 with $n=1$.

We conclude this section with a useful observation concerning orthogonal sums of 2hyperexpansive operators with invariant domains. It is easy to verify that if $T_{n}, n \geqslant$ 1, are 2-hyperexpansive operators (respectively, 2-isometries), then $T=\bigoplus_{n=1}^{\infty} T_{n}$ is a 2-hyperexpansive operator (respectively, 2-isometry). We are thus led to the following proposition.

Proposition 4.6. If $T_{n}, n \geqslant 1$, are 2-hyperexpansive operators (respectively, 2isometries) such that $T_{n} \mathcal{D}\left(T_{n}\right) \subseteq \mathcal{D}\left(T_{n}\right)$, then $T=\bigoplus_{n=1}^{\infty} T_{n}$ is a 2-hyperexpansive operator (respectively, 2-isometry) such that $T \mathcal{D}(T) \subseteq \mathcal{D}(T)$.

Proof. In both cases the following inequality holds $\left\|T_{n}^{2} f_{n}\right\|^{2} \leqslant 2\left\|T_{n} f_{n}\right\|^{2}$ for $f_{n} \in$ $\mathcal{D}\left(T_{n}\right)$, so the conclusion follows from Proposition $2.2(\mathrm{i})$.

\section{Spectra}

In this section we intend to describe the spectra of unbounded 2-hyperexpansive operators (see $[\mathbf{2}, \mathbf{3}, \mathbf{1 6}, \mathbf{2 0}]$ for the bounded case). We start with the following theorem.

Theorem 5.1. Let $T$ be a closed 2-hyperexpansive operator. Then

(i) $\operatorname{Sp}_{\mathrm{p}}(T) \subseteq\{z \in \mathbb{C}:|z|=1\}$,

(ii) $\mathbb{D} \subseteq \mathrm{Sp}(T) \backslash \mathrm{Sp}_{a p}(T)$ provided $T$ is 1-hyperexpansive and not 1-isometric,

(iii) $\mathbb{C} \backslash \overline{\mathbb{D}} \subseteq \operatorname{Sp}(T)$ provided $T_{[\infty]}$ is unbounded, and

(iv) $\mathbb{C} \backslash \overline{\mathbb{D}} \subseteq \operatorname{Sp}_{\mathrm{c}}(T)$ provided $\mathcal{D}^{\infty}(T)$ is dense in $\mathcal{H}$ and $T$ is unbounded.

Proof. (i) If $f \in \mathcal{D}(T),\|f\|=1$ and $T f=z f$ for some $z \in \mathbb{C}$, then

$$
\left(|z|^{2}-1\right)^{2}=\left\|T^{2} f\right\|^{2}-2\|T f\|^{2}+\|f\|^{2} \leqslant 0,
$$

so $|z|=1$.

(ii) It follows from Lemma 3.1 that $\mathbb{D} \subseteq \mathbb{C} \backslash \operatorname{Sp}_{\text {ap }}(T)$. Since the defect function $z \mapsto$ $\operatorname{ind}_{z} T \stackrel{\text { df }}{=} \operatorname{dim}[\mathcal{H} \ominus \mathcal{R}(T-z)]$ is constant on connected components of $\mathbb{C} \backslash \operatorname{Sp}_{\text {ap }}(T)$, the proof of the inclusion $\mathbb{D} \subseteq \operatorname{Sp}(T)$ is completed by showing that $\operatorname{ind}_{0} T>0$, or equivalently that 
$0 \in \operatorname{Sp}(T)$. Suppose, contrary to our claim, that $0 \notin \operatorname{Sp}(T)$. Since $T$ is 2 -hyperexpansive we have

$$
\left\|T^{2} f\right\|^{2}-2\|T f\|^{2}+\|f\|^{2} \leqslant 0, \quad f \in \mathcal{D}\left(T^{2}\right) .
$$

Substituting $f=T^{-2} h, h \in \mathcal{H}$, into the above inequality leads to

$$
\left\|T^{-2} h\right\|^{2}-2\left\|T^{-1} h\right\|^{2}+\|h\|^{2} \leqslant 0, \quad h \in \mathcal{H} .
$$

This means that $T^{-1} \in \boldsymbol{B}(\mathcal{H})$ is 2-hyperexpansive. It follows from [16, Lemma 1] that $\left\|T^{-1} h\right\| \geqslant\|h\|$ for $h \in \mathcal{H}$ and consequently that $\|f\| \leqslant\|T f\| \leqslant\|f\|$ for $f \in \mathcal{D}(T)$, which contradicts the assumption that $T$ is not 1-isometric.

(iii) Suppose that there exists $z \in \mathbb{C} \backslash \overline{\mathbb{D}}$ such that $(T-z)^{-1} \in \boldsymbol{B}(\mathcal{H})$. Since $\left(T_{[\infty]}-\right.$ $z)^{-1} \subseteq(T-z)^{-1}$, the operator $\left(T_{[\infty]}-z\right)^{-1}$ is bounded and, by Theorem 4.3 (ii), closed; consequently, the space $(T-z) \mathcal{D}^{\infty}(T)=\mathcal{D}\left(\left(T_{[\infty]}-z\right)^{-1}\right)$ is closed. It follows from Theorem 4.3 (iii) that the space $\mathcal{D}^{\infty}(T)$ is closed as well. By the closed graph theorem, the operator $T_{[\infty]}$ is bounded, which contradicts the assumption.

(iv) First we observe that $T_{[\infty]}$ is unbounded. Indeed, otherwise the inclusion $T_{[\infty]} \subseteq T$ and the equality $\overline{\mathcal{D}^{\infty}(T)}=\mathcal{H}$ imply, via Theorem 4.3 (ii), that $T=T_{[\infty]} \in \boldsymbol{B}(\mathcal{H})$, which contradicts our assumption. We now prove that $\mathbb{C} \backslash \overline{\mathbb{D}} \subseteq \operatorname{Sp}_{\mathrm{c}}(T)$. Take $z \in \mathbb{C} \backslash \overline{\mathbb{D}}$. It follows from Theorem 4.3 (iii) that

$$
\mathcal{H}=\overline{\mathcal{D}^{\infty}(T)}=\overline{\mathcal{R}\left(T_{[\infty]}-z\right)} \subseteq \overline{\mathcal{R}(T-z)} .
$$

However, by (i), $\mathcal{N}(T-z)=\{0\}$. Hence, according to (iii), $z \in \operatorname{Sp}_{\mathrm{c}}(T)$.

The spectrum of a 2-hyperexpansive operator which leaves its domain invariant can be described as follows (recall that according to [14, Proposition 2.1] a closed densely defined operator $T$ in $\mathcal{H}$ with invariant domain is in $\boldsymbol{B}(\mathcal{H})$ if and only if $\operatorname{Sp}(T) \neq \mathbb{C})$.

Proposition 5.2. If $T$ is an unbounded closed 2-hyperexpansive operator in $\mathcal{H}$ such that $\overline{\mathcal{D}(T)}=\mathcal{H}$ and $T \mathcal{D}(T) \subseteq \mathcal{D}(T)$, then

(i) $\operatorname{Sp}(T)=\mathbb{C}$,

(ii) $\mathbb{D} \subseteq \operatorname{Sp}_{\mathrm{r}}(T)$,

(iii) $\operatorname{Sp}_{\mathrm{p}}(T) \subseteq\{z \in \mathbb{C}:|z|=1\}$,

(iv) $\mathbb{C} \backslash \overline{\mathbb{D}} \subseteq \mathrm{Sp}_{\mathrm{c}}(T)$,

(v) $\operatorname{Sp}_{\text {ap }}(T)=\mathbb{C} \backslash \mathbb{D}$,

(vi) $\mathcal{R}(I-z T)=\mathcal{D}(T)$ for $z \in \mathbb{D}$, and

(vii) the spectral radius of $T$ calculated in $\boldsymbol{B}\left(\mathcal{D}(T),\|\cdot\|_{T, n}\right)$ is equal to 1 for $n \geqslant 1$.

Proof. Condition (i) follows either from Lemma 3.2 (iii) and Theorem 5.1 or from [14, Proposition 2.1]. Conditions (ii), (iii), (iv) and (v) can be deduced from Lemma 3.2 (iii) 
and Theorem 5.1 (because $\operatorname{Sp}(T) \backslash \operatorname{Sp}_{\mathrm{ap}}(T) \subseteq \operatorname{Sp}_{\mathrm{r}}(T), \operatorname{Sp}_{\mathrm{c}}(T) \subseteq \operatorname{Sp}_{\mathrm{ap}}(T)$ and the set $\operatorname{Sp}_{\text {ap }}(T)$ is closed). Condition (vi) is a direct consequence of Theorem 4.3 (iii).

(vii) It follows from* Lemma $4.1(\mathrm{i})$ and $(\mathrm{v})$ that $\left(\mathcal{D}(T),\|\cdot\|_{T, n}\right)$ is a Hilbert space and that $T$ is a bounded 2-hyperexpansive operator on $\left(\mathcal{D}(T),\|\cdot\|_{T, n}\right)$. Hence, $[\mathbf{2 0}$, Remark 3] completes the proof.

Corollary 5.3. Under the assumptions of Proposition 5.2 we have

(i) $\operatorname{Sp}\left(T^{*}\right)=\operatorname{Sp}_{\text {ap }}\left(T^{*}\right)=\mathbb{C}$,

(ii) $\mathbb{D} \subseteq \operatorname{Sp}_{\mathrm{p}}\left(T^{*}\right)$, and

(iii) $\mathbb{C} \backslash \overline{\mathbb{D}} \subseteq \operatorname{Sp}_{\mathrm{c}}\left(T^{*}\right)$.

Remark 5.4. It is well known that if $T=\bigoplus_{n=1}^{\infty} T_{n}$ is the orthogonal sum of hyponormal operators, then

$$
\operatorname{Sp}(T)=\overline{\bigcup_{n=1}^{\infty} \operatorname{Sp}\left(T_{n}\right)}
$$

This is no longer true for 2-hyperexpansive (what is more, for completely hyperexpansive) operators. Indeed, take a sequence of 2-hyperexpansive operators $T_{n} \in \boldsymbol{B}\left(\mathcal{H}_{n}\right), n \geqslant 1$, such that $\sup _{n \geqslant 1}\left\|T_{n}\right\|=\infty$ (cf. Example 6.4). Then, by Proposition 4.6, $T=\bigoplus_{n=1}^{\infty} T_{n}$ is an unbounded closed 2-hyperexpansive operator with invariant dense domain. Hence, according to [16, Lemma 1] and Proposition 5.2, we have

$$
\overline{\bigcup_{n=1}^{\infty} \operatorname{Sp}\left(T_{n}\right)} \subseteq \overline{\mathbb{D}} \subsetneq \mathbb{C}=\operatorname{Sp}(T)
$$

\section{All this for weighted shifts}

Let $\mathcal{K}$ be a separable Hilbert space with an orthonormal basis $\left\{e_{n}\right\}_{n=0}^{\infty}$ and let $\left\{\lambda_{n}\right\}_{n=0}^{\infty}$ be a sequence of positive numbers. The operator $S$ in $\mathcal{K}$ defined by

$$
\mathcal{D}(S)=\left\{f \in \mathcal{K}: \sum_{k=0}^{\infty}\left|\left\langle f, e_{k}\right\rangle\right|^{2} \lambda_{k}^{2}<\infty\right\}, \quad S f=\sum_{k=0}^{\infty}\left\langle f, e_{k}\right\rangle \lambda_{k} e_{k+1}, \quad f \in \mathcal{D}(S),
$$

is called a weighted shift with weights $\left\{\lambda_{n}\right\}_{n=0}^{\infty}$. The operator $S$ so defined is closed and the linear span of $\left\{e_{n}\right\}_{n=0}^{\infty}$ is a core of $S$ (cf. [10]; see also [18] for the bounded case). Moreover, $S$ is bounded if and only if the sequence $\left\{\lambda_{n}\right\}_{n=0}^{\infty}$ is bounded; if this happens, then $\|S\|=\sup _{n \geqslant 0} \lambda_{n}$. The weighted shift $U \in \boldsymbol{B}(\mathcal{K})$ with weights $\lambda_{n} \equiv 1$ (i.e. $U e_{n}=e_{n+1}$ for $n \geqslant 0$ ) is called the unilateral shift. Obviously $U$ is an isometry. All weighted shifts investigated in this section are considered with respect to the same orthonormal basis.

* It is also a consequence of Lemma 2.1 and the fact that $T$ is a closed operator on the Hilbert space $\left(\mathcal{D}(T),\|\cdot\|_{T, n}\right)$ (we only need to assume that $T$ is closed and $T \mathcal{D}(T) \subseteq \mathcal{D}(T)$ ). 
We begin this section by formulating basic properties of 2-hyperexpansive weighted shifts (cf. [2] for the description of completely hyperexpansive weighted shifts). The proof of the following fact is left to the reader.

Lemma 6.1. Let $S$ be a weighted shift with weights $\left\{\lambda_{n}\right\}_{n=0}^{\infty}$.

(i) $S$ is 2-hyperexpansive if and only if $\lambda_{n+1}^{2} \leqslant 2-\left(1 / \lambda_{n}^{2}\right)$ for $n \geqslant 0$.

(ii) If $S$ is a 2-isometry, then $\lambda_{0} \geqslant 1$ and $\lambda_{n}=\sigma_{n}\left(\lambda_{0}\right)$ for $n \geqslant 0$, where

$$
\sigma_{n}(\lambda)=\sqrt{\frac{1+(n+1)\left(\lambda^{2}-1\right)}{1+n\left(\lambda^{2}-1\right)}}, \quad n \geqslant 0, \quad \lambda \geqslant 1 .
$$

Conversely, for every $\lambda \geqslant 1$, the weighted shift $S_{\lambda}$ with the weight sequence $\left\{\sigma_{n}(\lambda)\right\}_{n=0}^{\infty}$ is a 2-isometry.

Notice that if $1 \leqslant \lambda \leqslant \lambda^{\prime}$, then $\sigma_{n}(\lambda) \leqslant \sigma_{n}\left(\lambda^{\prime}\right)$ for every $n \geqslant 0$.

Proposition 6.2. Let $S$ be a 2-hyperexpansive weighted shift with weights $\left\{\lambda_{n}\right\}_{n=0}^{\infty}$ and let $s \geqslant 0$. Then

(i) $S \in \boldsymbol{B}(\mathcal{K})$ and $\|S\|=\lambda_{0}$,

(ii) $\lambda_{k} \geqslant 1$ for $k \geqslant 0$,

(iii) $\lambda_{k} \geqslant \lambda_{k+1}$ for $k \geqslant 0$,

(iv) $\lim _{k \rightarrow \infty} \lambda_{k}=1$,

(v) $\lambda_{k} \leqslant \sigma_{k}\left(\lambda_{0}\right)$ for $k \geqslant 0$, where $\sigma_{k}\left(\lambda_{0}\right)$ are as in Lemma 6.1,

(vi) $\lambda_{k}=1$ for $k \geqslant s$ provided $\lambda_{s}=1$, and

(vii) $\lambda_{k}=1$ for $k \geqslant s$ provided $\lambda_{s}=\lambda_{s+1}$.

Proof. (iii) Suppose, contrary to our claim, that $\lambda_{k}<\lambda_{k+1}$ for some $k \geqslant 0$. Then, by Lemma 6.1 (i), we have

$$
0 \leqslant \lambda_{k}^{4}-2 \lambda_{k}^{2}+1<\lambda_{k}^{2} \lambda_{k+1}^{2}-2 \lambda_{k}^{2}+1 \leqslant 0,
$$

which is impossible.

Condition (i) follows from (iii), while (ii) is a consequence of (i) and Lemma 3.2 (iii) (because $\lambda_{k}=\left\|S e_{k}\right\|$ ).

(iv) It follows from (ii) and (iii) that the $\operatorname{limit} \lambda \stackrel{\mathrm{df}}{=} \lim _{k \rightarrow \infty} \lambda_{k}$ exists and $\lambda \geqslant 1$. According to Lemma 6.1 (i) we have $\lambda_{k+1}^{2}-2+\left(1 / \lambda_{k}^{2}\right) \leqslant 0$, so letting $k \rightarrow \infty$ yields $\lambda^{2}-2+\left(1 / \lambda^{2}\right) \leqslant 0$, which, in turn, gives us $\lambda=1$.

Applying an induction argument, one can deduce (v) from Lemma 6.1(i). Condition (vi) follows from (ii) and (iii).

(vii) Since $\lambda_{s}=\lambda_{s+1}$ and, by Lemma 6.1 (i), $\lambda_{s}^{2} \lambda_{s+1}^{2}-2 \lambda_{s}^{2}+1 \leqslant 0$ we conclude that $\lambda_{s}=1$. Applying (vi) completes the proof. 
Remark 6.3. Let $S$ and $S^{\prime}$ be 2-hyperexpansive weighted shifts with weight sequences $\left\{\lambda_{n}\right\}_{n=0}^{\infty}$ and $\left\{\lambda_{n}^{\prime}\right\}_{n=0}^{\infty}$, respectively. Then, by Lemma 6.1 (i), the weighted shifts with weight sequences $\left\{\lambda_{n+s}\right\}_{n=0}^{\infty}$ and $\left\{\lambda_{s, n}\right\}_{n=0}^{\infty}$, where $\lambda_{s, n}=\lambda_{n}$ for $n=0, \ldots, s-1$ and $\lambda_{s, n}=\min \left\{\lambda_{n}, \lambda_{n-s}^{\prime}\right\}$ for $n \geqslant s$, are 2-hyperexpansive $(s \geqslant 0)$. It follows from Lemma 6.1 (i) and Proposition 6.2 (i) that, after replacing the first weight $\lambda_{0}$ of $S$ by $\lambda_{0}+t, t \geqslant 0$, we get a 2-hyperexpansive weighted shift whose norm is equal to $\lambda_{0}+t$. Moreover, by parts (ii) and (v) of Proposition 6.2, the weights of $S$ are placed between weights of two extreme weighted shifts, the first of which is the unilateral shift $U$, while the other one is a 2 -isometry.

We are now in a position to exhibit examples of unbounded closed 2-isometries with invariant domains.

Example 6.4. Let $S_{\lambda}, \lambda \geqslant 1$, be the 2-isometry defined in Lemma 6.1 (ii). In view of Proposition 6.2 (i), $\left\|S_{\lambda}\right\|=\lambda$. Therefore, by Proposition 4.6, the operator $S=\bigoplus_{n=1}^{\infty} S_{n}$ is an unbounded closed 2-isometry with invariant dense domain.

The next result sheds more light on spectra of 2-hyperexpansive weighted shifts. Denote by $\mathcal{S}^{p}, p \geqslant 1$, the Schatten-von Neumann $p$-class of operators on $\mathcal{K}$.

Theorem 6.5. If $S$ is a 2-hyperexpansive weighted shift, then

(i) $S=U+\Delta_{S}$, where $\Delta_{S}$ is a compact operator which belongs to $\mathcal{S}^{p}$ for $p>1$; moreover, if $S$ is a 2-isometry which is not an isometry, then $\Delta_{S} \notin \mathcal{S}^{1}$, and

(ii) $\operatorname{Sp}_{\mathrm{p}}\left(S^{*}\right)=\mathbb{D}$.

Proof. (i) It suffices to show that $\left|\Delta_{S}\right| \stackrel{\mathrm{df}}{=}\left(\Delta_{S}^{*} \Delta_{S}\right)^{1 / 2}$ belongs to an appropriate ideal of operators (see [17] for more details). Let $\left\{\lambda_{n}\right\}_{n=0}^{\infty}$ be the weight sequence of $S$. Set $\delta_{n}=\lambda_{n}-1$ for $n \geqslant 0$. Then, by Proposition 6.2 (ii), $\Delta_{S}$ is a weighted shift with non-negative weights $\left\{\delta_{n}\right\}_{n=0}^{\infty}$, and, consequently, $\left|\Delta_{S}\right| e_{n}=\delta_{n} e_{n}$ for $n \geqslant 0$. In view of Proposition $6.2(\mathrm{iv}), \lim _{n \rightarrow \infty} \delta_{n}=0$, so the operator $\Delta_{S}$ is compact.

Since $\delta_{n}=\left(\left(\lambda_{n}^{2}-1\right) /\left(\lambda_{n}+1\right)\right)$ for $n \geqslant 0$, parts (ii) and (iii) of Proposition 6.2 imply that $\sum_{n=1}^{\infty} \delta_{n}^{p}<\infty$ if and only if $\sum_{n=1}^{\infty}\left(\lambda_{n}^{2}-1\right)^{p}<\infty(p>0)$. By Proposition $6.2(\mathrm{v})$ we have

$$
\sum_{n=1}^{\infty}\left(\lambda_{n}^{2}-1\right)^{p} \leqslant \sum_{n=1}^{\infty}\left(\sigma_{n}\left(\lambda_{0}\right)^{2}-1\right)^{p}, \quad p>0 .
$$

Notice also that

$$
\sigma_{n}\left(\lambda_{0}\right)^{2}-1=\frac{\lambda_{0}^{2}-1}{1+n\left(\lambda_{0}^{2}-1\right)}, \quad n \geqslant 1
$$

Combining (6.1) and (6.2) we conclude that $\Delta_{S} \in \mathcal{S}^{p}$ for every $p>1$. In the case in which $S$ is a 2 -isometry which is not an isometry (i.e. $\lambda_{0}^{2}-1>0$ ), (6.2) leads to $\Delta_{S} \notin \mathcal{S}^{1}$. 
(ii) We know that $\mathbb{D} \subseteq \operatorname{Sp}_{\mathrm{p}}\left(S^{*}\right)$ (cf. [2, Proposition 5]). Thus, by [22, $\left.\S 16\right]$, we only need to show that $\sum_{n=0}^{\infty}\left\|S^{n} e_{0}\right\|^{-2}=\infty$. According to Proposition $6.2(\mathrm{v})$ we have

$$
\begin{aligned}
\sum_{n=1}^{\infty}\left\|S^{n} e_{0}\right\|^{-2} & =\sum_{n=1}^{\infty} \frac{1}{\lambda_{0}^{2} \cdots \lambda_{n-1}^{2}} \\
& \geqslant \sum_{n=1}^{\infty} \frac{1}{\sigma_{0}\left(\lambda_{0}\right)^{2} \cdots \sigma_{n-1}\left(\lambda_{0}\right)^{2}}=\sum_{n=1}^{\infty} \frac{1}{1+n\left(\lambda_{0}^{2}-1\right)}=\infty
\end{aligned}
$$

which completes the proof.*

Referring to Theorem 6.5 (i), we establish 2-hyperexpansive weighted shifts $S$ such that $\Delta_{S} \in \mathcal{S}^{1}$.

Example 6.6. Take an arbitrary 2-hyperexpansive weighted shift $S$ with weights $\left\{\lambda_{n}\right\}_{n=0}^{\infty}$. Since $\lim _{n \rightarrow \infty}\left(\lambda_{n}-1\right)=0$, there exists a subsequence $\left\{k_{n}\right\}_{n=1}^{\infty}$ of $\{n\}_{n=0}^{\infty}$ such that $k_{1}=0$ and

$$
\sum_{n=1}^{\infty} \sum_{j=k_{2 n-1}}^{k_{2 n}}\left(\lambda_{j}-1\right)<\infty .
$$

Consider a new sequence

$$
\lambda_{k_{1}}, \ldots, \lambda_{k_{2}}, \lambda_{k_{3}}, \ldots, \lambda_{k_{4}}, \ldots, \lambda_{k_{2 n-1}}, \ldots, \lambda_{k_{2 n}}, \ldots,
$$

and denote its entries by $\lambda_{0}^{\prime}, \lambda_{1}^{\prime}, \lambda_{2}^{\prime}, \ldots$ One can check, using Lemma 6.1 (i) and Proposition 6.2 (iii), that the weighted shift $S^{\prime}$ with weights $\left\{\lambda_{n}^{\prime}\right\}_{n=0}^{\infty}$ is 2-hyperexpansive and that $\Delta_{S^{\prime}}$ is trace class.

We now show how to characterize 2-hyperexpansive operators with invariant domains by means of 2-hyperexpansive weighted shifts (see [8] for the case of bounded subnormal operators).

Proposition 6.7. If $T$ is an operator in $\mathcal{H}$ such that $T \mathcal{D}(T) \subseteq \mathcal{D}(T)$, then $T$ is 2hyperexpansive (respectively, 2-isometric) if and only $\mathcal{N}(T)=\{0\}$ and the weighted shift $S^{f}$ with the weight sequence

$$
\left\{\frac{\left\|T^{n+1} f\right\|}{\left\|T^{n} f\right\|}\right\}_{n=0}^{\infty}
$$

is 2-hyperexpansive (respectively, 2-isometric) for every $f \in \mathcal{D}(T) \backslash\{0\}$.

Proof. If $T$ is 2-hyperexpansive and $f \in \mathcal{D}(T) \backslash\{0\}$, then

$$
\left\|T^{n+2} f\right\|^{2}-2\left\|T^{n+1} f\right\|^{2}+\left\|T^{n} f\right\|^{2} \leqslant 0 .
$$

Dividing both sides of $(6.3)$ by $\left\|T^{n+1} f\right\|^{2}$, we get $\lambda_{f, n+1}^{2} \leqslant 2-\left(1 / \lambda_{f, n}^{2}\right)$ with $\lambda_{f, n}=$ $\left(\left\|T^{n+1} f\right\| /\left\|T^{n} f\right\|\right)$. By Lemma $6.1(\mathrm{i}), S^{f}$ is 2 -hyperexpansive. Likewise, we prove the converse implication as well as the case of 2-isometries.

* Using $[\mathbf{2 2}, \S 16]$, one can directly show that $\mathbb{D} \subseteq \operatorname{Sp}_{\mathrm{p}}\left(S^{*}\right)$. 
Applying Proposition 6.2 (iii), (vii) and (v), Lemma 6.1(ii) and Proposition 6.7, we get the following corollary.

Corollary 6.8. If $T$ is a 2-hyperexpansive operator in $\mathcal{H}$ with invariant domain and $f \in \mathcal{D}(T) \backslash\{0\}$, then

(i) $\left\{\left\|T^{n+1} f\right\| /\left\|T^{n} f\right\|\right\}_{n=0}^{\infty}$ is a monotonically decreasing sequence,

(ii) $\|T f\|^{2} \geqslant\|f\|\left\|T^{2} f\right\|$,

(iii) $\left\|T^{n} f\right\|=\|f\|$ for every $n \geqslant 0$ provided $\|T f\|^{2}=\|f\|\left\|T^{2} f\right\|$, and

$$
\frac{\left\|T^{n+1} f\right\|^{2}}{\left\|T^{n} f\right\|^{2}} \leqslant \frac{(n+1)\|T f\|^{2}-n\|f\|^{2}}{n\|T f\|^{2}-(n-1)\|f\|^{2}}
$$

for every $n \geqslant 0$.

Remark 6.9. Part (ii) of Corollary 6.8 reminds us of the definition of a paranormal operator, only the inequality is, in a sense, opposite. In fact, it remains true without assuming invariance of the domain of $T$. To be more precise, if $T$ is a 2-hyperexpansive operator in $\mathcal{H}$, then

$$
\|T f\|^{2} \geqslant \frac{1}{2}\left(\|f\|^{2}+\left\|T^{2} f\right\|^{2}\right) \geqslant\|f\|\left\|T^{2} f\right\|, \quad f \in \mathcal{D}\left(T^{2}\right) .
$$

If, moreover, $T$ is paranormal, then (6.4) implies that $\|T f\|^{2}=\|f\|\left\|T^{2} f\right\|$ for $f \in \mathcal{D}\left(T^{2}\right)$. This and the second inequality in (6.4) give us $\left\|T^{2} f\right\|=\|f\|$ for $f \in \mathcal{D}\left(T^{2}\right)$. Hence $\|T f\|^{2}=\|f\|\left\|T^{2} f\right\|=\|f\|^{2}$ for $f \in \mathcal{D}\left(T^{2}\right)$. This is an alternative proof of a part of Theorem 3.4. Notice also that part (iv) of Corollary 6.8 implies part (iv) of Lemma 3.2 in the case in which the operator in question leaves its domain invariant.

Our next goal is to show that some one-dimensional perturbations of 2-hyperexpansive weighted shifts remain 2-hyperexpansive (compare with Remark 3.6). Set $P_{0} h=\left\langle h, e_{0}\right\rangle e_{0}$ for $h \in \mathcal{K}\left(P_{0}\right.$ is the orthogonal projection of $\mathcal{K}$ onto $\left.\mathbb{C} e_{0}\right)$.

Proposition 6.10. Let $S$ be a 2-hyperexpansive (respectively, 2-isometric) weighted shift with weights $\left\{\lambda_{n}\right\}_{n=0}^{\infty}$ and let $z \in \mathbb{C}$. Then

(i) $r_{\lambda_{0}, \lambda_{1}} \stackrel{\text { df }}{=} \frac{1}{2}\left(2-\lambda_{0}^{2}+\lambda_{0} \sqrt{\lambda_{0}^{2}-4 \lambda_{1}^{2}+4}\right) \geqslant 0$; moreover, $r_{\lambda_{0}, \lambda_{1}}=0$ if and only if $\lambda_{0} \geqslant \sqrt{2}$ and $\lambda_{1}=\sigma_{1}\left(\lambda_{0}\right)$,

(ii) the operator $S+z P_{0}$ is 2-hyperexpansive (respectively, 2-isometric) if and only if $|z|^{2} \leqslant r_{\lambda_{0}, \lambda_{1}}$ (respectively, $z=0$ or $\left.|z|^{2}=r_{\lambda_{0}, \lambda_{1}}\right)$.

Proof. (i) It follows from Lemma 6.1 (i) that $\vartheta_{\lambda_{0}, \lambda_{1}} \stackrel{\mathrm{df}}{=} 2 \lambda_{0}^{2}-\lambda_{0}^{2} \lambda_{1}^{2}-1 \geqslant 0$, hence that $\lambda_{0}^{2}-4 \lambda_{1}^{2}+4=\lambda_{0}^{-2}\left(\left(2-\lambda_{0}^{2}\right)^{2}+4 \vartheta_{\lambda_{0}, \lambda_{1}}\right) \geqslant 0$ and finally that

$$
2 r_{\lambda_{0}, \lambda_{1}}=\left(2-\lambda_{0}^{2}\right)+\sqrt{\left(2-\lambda_{0}^{2}\right)^{2}+4 \vartheta_{\lambda_{0}, \lambda_{1}}} \geqslant\left(2-\lambda_{0}^{2}\right)+\left|2-\lambda_{0}^{2}\right| \geqslant 0 .
$$


If $\lambda_{1}=\sigma_{1}\left(\lambda_{0}\right)\left(\Leftrightarrow \vartheta_{\lambda_{0}, \lambda_{1}}=0\right)$, then, by $(6.5), r_{\lambda_{0}, \lambda_{1}}=0$ for $\lambda_{0} \geqslant \sqrt{2}$ and $r_{\lambda_{0}, \lambda_{1}}>0$ for $\lambda_{0}<\sqrt{2}$. In the case where $\lambda_{1} \neq \sigma_{1}\left(\lambda_{0}\right)\left(\Leftrightarrow \vartheta_{\lambda_{0}, \lambda_{1}}>0\right)$, (6.5) yields $r_{\lambda_{0}, \lambda_{1}}>0$.

(ii) Set $T=S+z P_{0}$. It is a simple matter to verify that if $\left\{\alpha_{n}\right\}_{n=0}^{\infty} \in \ell^{2}$, then

$$
\begin{aligned}
\left\|T^{2} f\right\|^{2}-2\|T f\|^{2}+\|f\|^{2}=\left|\alpha_{0}\right|^{2}\left(|z|^{4}+|z|^{2}\left(\lambda_{0}^{2}-2\right)+\left(\lambda_{0}^{2} \lambda_{1}^{2}-2 \lambda_{0}^{2}+1\right)\right) \\
+\sum_{n=1}^{\infty}\left|\alpha_{n}\right|^{2}\left(\lambda_{n}^{2} \lambda_{n+1}^{2}-2 \lambda_{n}^{2}+1\right), \quad f=\sum_{n=0}^{\infty} \alpha_{n} e_{n} .
\end{aligned}
$$

According to Lemma 6.1 (i), $\lambda_{n}^{2} \lambda_{n+1}^{2}-2 \lambda_{n}^{2}+1 \leqslant 0$ for $n \geqslant 0$. Hence, by (6.6), the operator $T$ is 2-hyperexpansive if and only if

$$
|z|^{4}+|z|^{2}\left(\lambda_{0}^{2}-2\right)+\left(\lambda_{0}^{2} \lambda_{1}^{2}-2 \lambda_{0}^{2}+1\right) \leqslant 0 .
$$

In view of (6.5), the inequality (6.7) is equivalent to $|z|^{2} \leqslant r_{\lambda_{0}, \lambda_{1}}$. The same reasoning applies to the case of 2-isometries.

Referring to Proposition 6.10 (and Remark 6.3), consider an arbitrary 2-hyperexpansive weighted shift $S$. Leaving the weights $\lambda_{1}, \lambda_{2}, \ldots$ unchanged, one can show (making use of (i) and (6.5)) that $\lim _{\lambda_{0} \rightarrow \infty} r_{\lambda_{0}, \lambda_{1}}=2-\lambda_{1}^{2}>0$. Hence, by (ii), for every $z \in \mathbb{C}$ with $|z|<\sqrt{2-\lambda_{1}^{2}}$, there exists $R>0$ such that $S+z P_{0}$ is 2-hyperexpansive for $\lambda_{0} \geqslant R$. Notice that $\left\|S+z P_{0}\right\|=\sqrt{\lambda_{0}^{2}+|z|^{2}} \geqslant \lambda_{0}$.

We conclude the paper with the following observation.

Proposition 6.11. If $S$ is a closed bilateral weighted shift with positive weights $\left\{\lambda_{n}\right\}_{n=-\infty}^{\infty}$ which is 2-hyperexpansive, then $S$ is unitary, i.e. $\lambda_{n} \equiv 1$.

Proof. Let $\left\{e_{n}\right\}_{n=-\infty}^{\infty}$ be an orthonormal basis with respect to which $S$ is a bilateral weighted shift and let $\mathcal{E}$ be the linear span of $\left\{e_{n}\right\}_{n=-\infty}^{\infty}$. Applying Proposition 3.5 (i) to the operator $T=\left.S\right|_{\mathcal{E}}$, we conclude that $S=\bar{T}$ is unitary.

Acknowledgements. The research carried out by J.S. was supported by KBN grant no. 2 P03A 00417.

\section{References}

1. J. Agler and M. Stankus, $m$-isometric transformations of Hilbert space, Part I, Integ. Eqns Operat. Theory 21 (1995), 383-429.

2. A. Athavale, On completely hyperexpansive operators, Proc. Am. Math. Soc. 124 (1996), 3745-3752.

3. A. Athavale and V. M. Sholapurkar, Completely hyperexpansive operator tuples, Positivity 3 (1999), 245-257.

4. T. Furuta, On the class of paranormal operators, Proc. Japan. Acad. 43 (1967), 594-598.

5. V. I. Istrăţ̧ESCU, Introduction to linear operator theory, in Pure and applied mathematics, vol. 65 (Marcel Dekker, New York, 1981).

6. V. Istrătescu, T. Saitô and T. Yoshino, On a class of operators, Tôhoku Math. J. 18 (1966), 410-413.

7. J. JANAS, On unbounded hyponormal operators, Ark. Math. 27 (1989), 273-281. 
8. A. LAmbert, Subnormality and weighted shifts, J. Lond. Math. Soc. 14 (1976), 476-480.

9. G. LAssner, Topological algebras of operators, Rep. Math. Phys. 3 (1972), 276-293.

10. W. Mlak, The Schrödinger type couples related to weighted shifts, Univ. Iagell. Acta Math. 27 (1988), 297-301.

11. Y. OKazAki, Boundedness of closed linear operator $T$ satisfying $R(T) \subset D(T)$, Proc. Japan. Acad. 62 (1986), 294-296.

12. S. ÔtA, Closed derivations in $C^{*}$-algebras, Math. Annln 257 (1981), 239-250.

13. S. ÔTA, Closed linear operators with domain containing their range, Proc. Edinb. Math. Soc. 27 (1984), 229-233.

14. S. ÔtA, Unbounded nilpotents and idempotents, J. Math. Analysis Appl. 132 (1988), 300-308.

15. S. ÔtA AND K. SchmüDGen, On some classes of unbounded operators, Integ. Eqns Operat. Theory 12 (1989), 211-226.

16. S. Richter, Invariant subspaces of the Dirichlet shift, J. Reine Angew. Math. 386 (1988), 205-220.

17. J. R. RINGROSE, Compact non-self-adjoint operators, van Nostrand Reinhold Mathematical Studies, vol. 35 (1971).

18. A. L. ShiElds, Weighted shift operators and analytic function theory, Mathematical Surveys, no. 13, pp. 49-128 (American Mathematical Society, Providence, RI, 1974).

19. S. Shimorin, Wold-type decompositions and wandering subspaces for operators close to isometries, J. Reine Angew. Math. 531 (2001), 147-189.

20. V. M. Sholapurkar and A. Athavale, Completely and alternatingly hyperexpansive operators, J. Operat. Theory 43 (2000), 43-68.

21. J. G. Stampfli, Hyponormal operators, Pac. J. Math. 12 (1962), 1453-1458.

22. J. Stochel And F. H. SzAfraniec, On normal extensions of unbounded operators, III, Publ. RIMS Kyoto Univ. 25 (1989), 105-139.

23. J. Stochel And F. H. Szafraniec, $\mathcal{C}^{\infty}$-vectors and boundedness, Ann. Polon. Math. 66 (1997), 223-238.

24. F. H. SzAFraniec, Kato-Protter type inequalities, bounded vectors and the exponential function, Ann. Polon. Math. 51 (1990), 303-312. 